\title{
Thromboembolic Stroke: A Rare Complication Associated with Peri-Procedural Management of an Epidural Steroid Injection
}

Andrew J. Linn, MD¹, Cecil DeSilva, MD², and Christine Peeters-Asdourian, MD³

From: ${ }^{1}$ Beth Israel Deaconess Medical Center, Department of Anesthesiology and Pain Medicine, Harvard Medical School, Boston MA; ${ }^{2}$ Rush University Medical Center, Chicago, IL; ${ }^{3}$ Arnold Pain Management Center, Beth Israel Deaconess Medical Center, Harvard Medical School, Boston, MA.

Address correspondence: Andrew J. Linn, MD 21 Revere St \#5 Boston, MA 02114

E-mail: andrewjlinn@gmail.com

Disclaimer: This was previously shown as an abstract at the Spring 2007 ASRA Meeting: Reg Anesth Pain Med 2007; 32: A-30. There was no external funding in the preparation of this manuscript. Conflict of interest: None.

Manuscript received: $04 / 13 / 2008$ Revised manuscript received: $10 / 07 / 2008$

Accepted for publication: $11 / 10 / 2008$
Background: Little attention has been afforded to the potential adverse sequelae of withholding anticoagulation therapy in a patient after neuraxial interventions.

Objective: Presenting a case of thromboembolic stroke in a patient after holding warfarin for a lumbar epidural steroid injection, previously unreported in published literature. The discussion that follows reviews the guidelines available to reduce the risk of thromboembolic events in anticoagulated patients in the periprocedural period.

Case Report: An 81-year-old female with radicular pain secondary to spinal stenosis had been seen on 5 previous occasions for lumbar epidural steroid injections. Prior to each procedure warfarin was held for 5-7 days with demonstrable reversal of anticoagulation to within the safe limits set for neuraxial techniques by the American Society of Regional Anesthesia and Pain Medicine. On the morning following the sixth injection the patient was admitted to the hospital for new onset slurred speech and left-sided hemiparesis.

A computed tomography scan established an acute, localized infarct in the distribution of the right middle cerebral artery. Her symptoms were non-reversible and permanent.

Conclusions: Thromboembolism is a potentially devastating complication associated with atrial fibrillation. Twenty percent of thromboembolic events in patients with atrial fibrillation are fatal, and greater than $50 \%$ result in permanent disability. While thromboembolic events following a brief period of normalization of coagulation for interventions appear rare, so is the incidence of epidural hematomas. Considering the high mortality and permanent rate of disability with thromboembolic events associated with atrial fibrillation, perhaps it is time to balance our focus on the complications of withholding anticoagulation with those of bleeding.

Key words: Stroke, atrial fibrillation, epidural steroid injection complication, anticoagulation

Pain Physician 2009;12:1:159-162

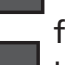

ortsaimedatreducing theincidence ofepidural hematomas in the anticoagulated patient after neuraxial interventions have garnered great interest over the past few years; considerably less attention has been afforded the potential adverse sequelae of withholding anticoagulation therapy. This case illustrates one such complication, resulting from subtherapeutic anticoagulation in a patient with atrial fibrillation. The discussion that follows will review the guidelines available to reduce the risk of thromboembolic events in anticoagulated patients in the periprocedural period. 


\section{Case Description}

An 81-year-old female with a history of radicular pain secondary to spinal stenosis had been seen on 5 previous occasions over a period of 19 months in the pain clinic for lumbar epidural steroid injections (LESIs). Significant past medical history included atrial fibrillation, previous tissue mitral valve replacement, hypertension, and Alzheimer's dementia. Her medications included acetaminophen, warfarin, lisinopril, propranolol, and Sinemet. She had no previously documented transient ischemic attacks (TIA) or cerebrovascular accident (CVA). Her initial description was non-constant pain of the axial low lumbar spine radiating to the bilateral buttocks, and periodically down the bilateral lower extremities to the popliteal fossa. Magnetic resonance imaging (MRI) confirmed spinal stenosis most severe at the L4-L5 level. The patient's previous LESIs had provided significant pain relief for periods ranging from 4 to 8 weeks. Prior to each epidural steroid injection warfarin was held for 5 to 7 days, and coagulation studies were obtained to demonstrate the reversal of anticoagulation to within the safe limits set for neuraxial techniques by the American Society of Regional Anesthesia and Pain Medicine (ASRA) (1). Prior to her first LESI, the risks and benefits of the procedure were discussed, including potential morbidity associated with discontinuing her anticoagulation. Further, the patient received approval from her managing physician to discontinue her warfarin for the procedures. During her treatment course, we also discussed with both the patient and her daughter the possibility of surgical intervention for her spinal stenosis; the patient refused to consider surgery, a position that was supported by her daughter.

The patient returned for a routine clinic visit having held her warfarin for 7 days in anticipation of a repeat LESI. At that time she was found to be disoriented, uncooperative, and hostile to pain clinic staff; symptoms she had regularly demonstrated. The patient's daughter, her healthcare proxy, was contacted and told that the patient was not appropriate for the procedure considering the acute exacerbation of her chronic dementia. Instructions were given to continue to hold the warfarin, and return to clinic in 2 days to perform the procedure. The patient returned to clinic after the prescribed time and was alert and oriented $\mathrm{x}$ 2 , pleasant, and continued to have normalized coagulation studies (INR 1.1, PT 13.0, PTT 18.1). An atraumatic LESI was performed with $3 \mathrm{~mL}$ of $1 \%$ lidocaine and $80 \mathrm{mg}$ of methylprednisolone acetate injected into the L5-S1 epidural space under fluoroscopic guidance and confirmed with radiographic contrast. No sedation was given. The patient tolerated the procedure without complaint, and had neither significant hemodynamic changes nor new neurologic deficits prior to leaving the clinic 35 minutes after the injection. She resumed her warfarin the same day, 8 hours after the procedure.

The first morning post-procedure the patient was admitted to the hospital via the emergency department for new onset slurred speech and left-sided hemiparesis. A CT scan done upon hospital admission demonstrated an acute, localized infarct in the distribution of the right middle cerebral artery. Her left hemiparesis, left-side neglect, and dysarthria continued beyond her discharge to a rehabilitation hospital.

\section{Discussion}

Atrial fibrillation (AF) is the most common arrhythmia encountered in clinical practice, with an estimated prevalence of 2.3 million in the United States. It is primarily a disease of the elderly: $70 \%$ of patients with AF are over the age of 65 , and AF is found in $9 \%$ of those older than 80 years of age (2). As such, our aging population will cause continued increases in $A F$, with anticipation of a prevalence of 5.6 million by the year 2050 (2).

Thromboembolism is a potentially devastating complication associated with AF. One-sixth of all ischemic strokes in patients over 60 years of age is attributed to AF (3). Strokes are more severe and transient ischemic attacks are longer in duration when secondary to AF than when caused by carotid disease, another leading cause of thromboemboli $(4,5)$. However, not all patients with AF are at the same risk for embolic events. One model for estimation of the risk of ischemic stroke in patients with nonvalvular AF is the CHADS-2 score. One point is given for each Congestive heart failure, Hypertension, Age > 75 years, Diabetes mellitus, and for a history of Stroke, 2 points are given. The CHADS-2 estimates the annual risk of a thromboembolic event in patients not using prophylaxis therapy; scores of $0-2,3-4$, and 5-6 represent an annual risk of $2-4 \%, 6-9 \%$, and $13-18 \%$, respectively (6). There are several guidelines used to direct anticoagulation therapy in patients with $\mathrm{AF}$, most using similar risk stratification as those drawn-out in CHADS-2. The American College of Cardiology (ACC)/American Heart Association (AHA)/European Society of Cardiology (ESC) joint guidelines recommend a goal Interna- 
tional Normalized Ratio (INR) of 2-3 as prophylaxis against thromboembolic events in patients with $A F$, and recommend even higher therapeutic goals (INR of 3-4) in patients with prosthetic heart valves (7); the American College of Chest Physicians (ACCP) has similar guidelines (8).

Maintaining recommended levels of anticoagulation during many of the procedures of interventional pain medicine would place our patients at undue risk of bleeding complications. For this reason specific guidelines have been developed regarding how long patients should hold anticoagulation and when to restart medications prior to and after regional anesthesia and pain procedures. Less attention is paid to the potential complications of withholding oral anticoagulation, which can be severe. Twenty percent of thromboembolic events in patients with AF are fatal, and greater than $50 \%$ result in permanent disability (6). There are limited data on the incidence of thromboembolism and stroke during discontinuation of longterm anticoagulation in the perioperative period. A pooled analysis of 31 "generally poor" studies found an overall incidence of $1.6 \%$ and $0.4 \%$ for thromboembolic events and cerebrovascular accidents, respectively, in patients holding long-term anticoagulation in the perioperative period (9). These data were not stratified by risk factor.

While there is no consensus on the management of anticoagulation in the perioperative/periprocedural period, there are several strategies to minimize the risks of fully reversing oral anticoagulation in the perioperative or periprocedural period. These bridging strategies involve either unfractionated heparin (UFH) or low molecular weight heparin (LMWH) therapy while oral vitamin $\mathrm{K}$ antagonists are held and the INR is normalized (10). The use of LMWH as an anticoagulation bridge is feasible, due to its predictable activity and elimination (11). Ease of home self-administration makes this option convenient and cost effective when compared to continuous infusions of UFH. Jaffer (12) describes strategies for managing oral anticoagulation in low, moderate, or high-risk patients with atrial fibrillation in the perioperative period, based on CHADS-2 scoring system. The author suggests bridging therapy for a CHADS-2 score of 3 or higher, indicating a $6 \%$ or greater annual (not perioperative) probability for stroke. Separately the ACCP and ACC/AHA have produced similar guidelines. While most guidelines agree on the value of providing LMWH or UFH in the peri- operative period to high-risk patients, there is some discrepancy on the management of intermediate and low-risk patients $(10,12-13)$. Unfortunately there are no randomized control trials nor large-scale prospective cohort studies that evaluate bridging strategies, rather retrospective, small cohort studies, and expert opinion form the basis of these guidelines.

The ACCP recommend in low-risk patients discontinuing warfarin therapy 4 days before the procedure. They make no specific guidelines regarding pre-procedure bridging in low-risk patients, but recommend beginning either UFH or LMWF post-procedure until warfarin becomes therapeutic. In intermediate-risk patients they recommend stopping warfarin 4 days pre-operatively, and bridging therapy with prophylactic doses of LMWH pre- and post-procedure until INR becomes therapeutic. In high-risk patients the recommendation is to discontinue warfarin 4 days before procedure, and bridging with full-strength LMWH before and after the procedure until INR levels become therapeutic (10). ASRA guidelines are to allow 12 hours after the last prophylactic dose of LMWH before neuraxial injections, and 24 hours after a full dose. They also recommend waiting 12 to 24 hours after neuraxial interventions before resuming $\mathrm{LMWH}$, depending on the dose, and if the injection was traumatic (1).

Coordination between the pain physician and the physician managing anticoagulation on the appropriateness of a brief period of normalization of coagulation status is crucial. Many of the physicians prescribing anticoagulants have a limited understanding of the procedures done in the pain clinic, making the unilateral evaluation of the risks and benefits of discontinuing anticoagulation for neuraxial procedures difficult and incomplete. As pain physicians, an understanding of simple guidelines will help us to decide, pre-emptively, on the appropriateness in the discontinuing anticoagulation therapies based on established risk factors. If a decision that the benefit of stopping the therapy appears to outweigh the potential for harm from a thromboembolic event, further seeking the opinion of whomever is managing the medications on the appropriateness of a brief period of normalization of coagulation, and the feasibility of bridging strategies, is warranted. Additionally, an understanding of the risk factors of temporarily discontinuing anticoagulation will enable a more in-depth explanation to our patients, ultimately allowing them to make more informed decisions regarding their healthcare. 


\section{References}

1. Horlocker TT, Wedel DJ, Benzon H, Brown DL, Enneking FK, Heit JA, Mulroy MF, Rosenquist RW, Rowlingson J, Tryba $M$, Yuan CS. Regional anesthesia in the anticoagulated patient: Defining the risks (the second ASRA Consensus Conference on Neuraxial Anesthesia and Anticoagulation). Reg Anesth Pain Med 2003; 28:172-197.

2. Gage BF, Waterman AD, Shannon W, Boechler M, Rich MW, Radford MJ. Validation of clinical classification schemes for predicting stroke: Results from the National Registry of Atrial Fibrillation. JAMA 2001;285:2864-2870.

3. Go AS, Hylek EM, Phillips KA, Chang Y, 8 . Henault LE, Selby JV, Singer DE. Prevalence of diagnosed atrial fibrillation in adults: National implications for rhythm management and stroke prevention: The Anticoagulation and Risk Factors in Atrial Fibrillation (ATRIA) Study. JAMA 2001; 285:2370-2375.

4. Anderson DC, Kappelle LJ, Eliasziw M, Babikian VL, Pearce LA, Barnett HJ. Occurrence of hemispheric and retinal ischemia in atrial fibrillation compared withcarotid stenosis. Stroke 2002; 33:1963-1967.
5. Harrison MJ, Marshall J. Atrial fibrillation, TIAs and completed strokes. Stroke 1984; 15:441-442.

6. Atrial Fibrillation Investigators. Risk factors for stroke and efficacy of antithrombotic therapy in atrial fibrillation. Analysis of pooled data from five randomized controlled trials. Arch Intern Med 1994; 154:1449-1457.

7. American College of Cardiology, American Heart Association, European Society of Cardiology. ACC/AHA/ESC guidelines for the management of patients with atrial fibrillation. J Am Coll Cardiol 2001; 38:1266i-lxx.

8. Hirsh J, Dalen J, Guyatt G. The sixth (2000) ACCP guidelines for antithrombotictherapy for prevention and treatment of thrombosis. American College of Chest Physicians. Chest 2001; 119:1S2S.

9. Dunn AS, Turpie AG. Perioperative management of patients receiving oral anticoagulants: A systematic review. Arch Intern Med 2003; 163:901-908.

10. Ansell J, Hirsh J, Poller L, Bussey H, Jacobson A, Hylek E. The pharmacology and management of the vitamin $\mathrm{K}$ antagonists: The Seventh ACCP Confer- ence on Antithrombotic and Thrombolytic Therapy. Chest. 2004; 126:204S233 S.

11. Kovacs MJ, Kearon C, Rodger M, Anderson DR, Turpie AG, Bates SM, Desjardins L, Douketis J, Kahn SR, Solymoss $S$, Wells PS. Single-arm study of bridging therapy with low-molecular-weight heparin for patients at risk of arterial embolism who require temporary interruption of warfarin. Circulation 2004; 110:1658-1663.

12. Jaffer AK. Anticoagulation management strategies for patients on warfarin who need surgery. Cleve Clin J Med 2006; 73:S100-S105.

13. Bonow RO, Carabello B, de Leon AC, Edmunds LH, Fredderly BJ, Freed MD, Gaasch WH, McKay CR, Nishimura RA, O'Gara PT, O'Rourke RA, Rahimtoola SH, Ritchie JL, Cheitlin MD, Eagle KA, Gardner TJ, Garson A, Gibbons RJ, Russell RO, Ryan TJ, Smith SC. Guidelines for the management of patients with valvular heart disease: Executive summary. A report of the American College of Cardiology/American Heart Association Task Force on Practice Guidelines. Circulation 1998; 98:1949-1984. 